\title{
Publisher Correction: Midbrain activity can explain perceptual decisions during an attention task
}

James P. Herman (D), Leor N. Katz and Richard J. Krauzlis (D)

Correction to: Nature Neuroscience https://doi.org/10.1038/s41593-018-0271-5, published online 26 November 2018.

In the version of this article initially published online, Fig. 1f,g contained errors: in panel G, the axes for $d$ ' were erroneously displayed as vertical rather than horizontal; and the labels "Monkey" and "Model" in panel F should have been vertically oriented rather than diagonally. The error has been corrected in the PDF and HTML versions of this article.
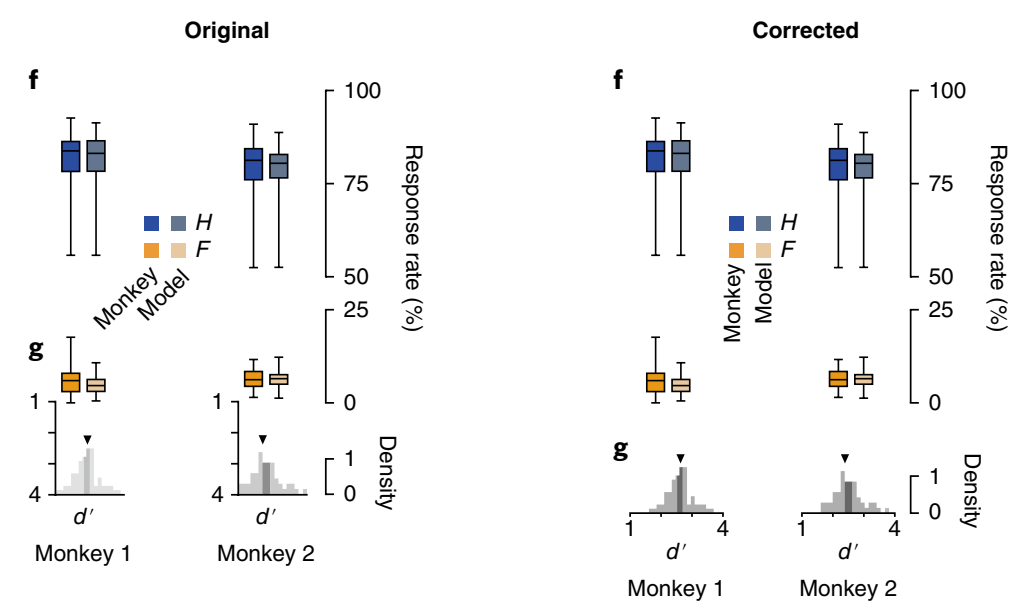

Figure 1f and $\mathrm{g}$ | Original and Corrected

Published online: 15 January 2019

https://doi.org/10.1038/s41593-018-0319-6

\section{Publisher Correction: Motor primitives in space and time via targeted gain modulation in cortical networks}

Jake P. Stroud D, Mason A. Porter, Guillaume Hennequin and Tim P. Vogels $\mathbb{D}$

Correction to: Nature Neuroscience https://doi.org/10.1038/s41593-018-0276-0, published online 26 November 2018.

In the version of this article initially published, in the PDF, equations (2) and (4) erroneously displayed a curly bracket on the right hand side of the equation. This should not be there. The errors have been corrected in the PDF version of the article. The equations appear correctly in the HTML.

In the supplementary information originally posted, corrupted characters appeared in the Supplementary Figure legends: in Supplementary Figs. 3 and 4, all instances of " $\gamma^{(\mathrm{mm})}$ " should have been $l$; in Supplementary Fig. 5, the first and second " $\partial^{(\mathrm{cm})}$ should have been $x$, and the " $\partial$ " in panel $\mathbf{i}$ should have been ' $m$ '. These errors have been corrected online. The legends appear correctly in the PDF. 